\begin{tabular}{c} 
International Journal of Engineering \& Technology, 7 (3) (2018) 1130-1135 \\
International Journal of Engineering \& Technology \\
SPC \\
Website: $\begin{array}{c}\text { www. sciencepubco.com/index.php/IJET } \\
\text { doi: } 10.14419 / \text { ijet. } 7 \text { i3.12557 } \\
\text { Research paper }\end{array}$ \\
\hline
\end{tabular}

\title{
FPGA-based color enhancement algorithm for thermal object characterization
}

\author{
Chan Su Park ${ }^{1}$, Hi Seok Kim ${ }^{1}$ * \\ ${ }^{I}$ Department of Electronics Engineering Cheongju University \\ *Corresponding author E-mail: khs8391@cju.ac.kr
}

\begin{abstract}
Thermal imaging is used in numerous applications, especially in security, medical and other industry which requires a non-contact temperature measurement. This proposed algorithm improves the thermal image and makes more visible the separation of the sampled object from its background. The extracted image is produced by the following techniques: the pre-processing techniques are the combination of K-means clustering, and inverted Otsu method; canny edge detection and color mapping are used for highlighting the necessary characteristics of the sampled thermal image. The experimental results of this proposed algorithm show significant distinguishable features in terms of edge and color enhancement. It outperforms the other color correction method in terms of processing time, and the implementation reduced the resource utilization. Moreover, it minimizes the misclassified pixel in different noise variance. This work is synthesized with Xilinx Zync 7000 ZED ZC702.
\end{abstract}

Keywords: Color Mapping; Inverted Otsu Method; K-Means Clustering; Object Boundary; Thermal Image.

\section{Introduction}

Thermal imaging is a method to convert the invisible radiation pattern effect of an object into visible images for feature extraction and analysis. It was developed for military purposes but these days gained a wide application in various fields such as aerospace, agriculture, medicine, and non-destructive testing fields. It can be applied in all fields where temperature variance is important for evaluation, diagnosis, or analysis of a process or product. The process is to identify the invisible infrared radiation effects, and making it visible to human eyes [1-2]. An ordinary camera is possible to record the data images event at a distance in real time, however, deterrent effects cannot be avoided such as noise which reflects lighting effect especially when s regular RGB cameras are used [3]. Therefore, thermal imagery equipment is used. The basic concepts in thermal imaging technology are based on the relationship between the object's characteristics and the intensity of infrared radiation effects and includes the temperature of objects and its surroundings. The most valuable application of thermal imaging is to detect any leak problems and in an industry that involves thermal anomalies. Figure 1 shows an example of thermal imaging, it shows that the compressor is already on over-heated stage or showing an abnormal heat.

A thermal imager equipment alone is enough, it only defines the image preliminary characteristics, because sample images have unnecessary characteristics like with high noise, low contrast, background interferences etc. [4]. Implementing an appropriate algorithm to facilitate these existing drawbacks. This paper shows an alternative algorithm to identify the optimum thermal image characteristics and certain parameters related to thermal imaging and how to diagnose these faults.

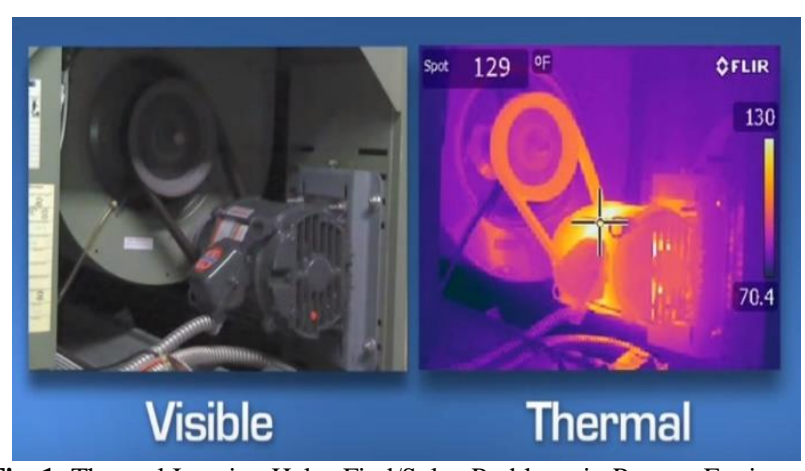

Fig. 1: Thermal Imaging Helps Find/Solve Problems in Process Equipment with Abnormal Heat Flow. (Www.Thermalimagercamera.Com).

The main concept of this proposed algorithm is to solve the existing drawback of Otsu technique and K-means clustering method. In Otsu method, the assumption on the histogram is to have bimodal distribution and to have a deep and pointed notch between two peaks. However, if the of the object area is smaller than the background, it will result in a non-modality histogram [5]. Moreover, the gray level of the histogram is degraded if the variances of the object and the background intensities are larger than the mean difference, resulting to an incorrect threshold in the segmentation error On the other hand, K-means clustering uses a local optimal method which does not require computing a gray level histogram before running, and it is appropriate for multilevel thresholding to compare in Otsu method. However, it is difficult to predict the k-value, and it does not work properly in the global clustering mode. Moreover, with different initial partitions, it will result in a different final cluster. Additionally, and, it will not work well in clusters of different size and density. Furthermore, this paper proposed a histogram clustering threshold based on color correction algorithm [6] using an iterative threshold, the minimum histogram difference is computed and correct the target object. Achieving better thermal object 
characterizations in terms of shape information, edge images. Lastly, this is implemented in an FPGA-based platform which eliminates unnecessary hardware and minimizes the processing time [78].

The rest of this paper is organized as follows. In Section 2, discussion of some related works to the proposed algorithm such as the basis of thermal concept, thermal images characteristics and existing works based on Otsu method, K-means, color mapping and other techniques in thermal imaging. Section 3 presents and describes the proposed algorithm, it includes implementation details. Experimental results and discussion are provided in Section 4. Finally, in Section 5, concludes this work.

\section{Review of related works}

In this section, some related works that are relevant to the proposed algorithm like the K-means clustering scheme [9-11], Otsu method [12-14] and color mapping techniques [15-18] for image enhancement are discussed. Material investigations are also involved, which called as nondestructive testing techniques. This method is defined as the method for examining a part, material, images, or system without impairing the original object [19-20], resulting in high reliability and cost-effective method.

Thermal imaging or thermal video are techniques under infrared imaging science. The basic concept is to improve an object or image to make it more visible to the naked human eye. Moreover, it helps to analyze by detecting the objects' infrared radiation pattern and its existing problems. The thermal imaging theory consists of three independent processes such as focused thermal radiation on the objects and its surroundings, the effect of the temperature pattern over the affected area and the physical property of the object, and changes in the communication to the human eye. The range of radiation detection of thermal imaging cameras in the electromagnetic spectrum is around 9-14 $\mu \mathrm{m}$ and the produced image is called as the thermal image or thermograms. A number of characteristics are considered in observing or, detecting an object in a background, these necessary elements are target-to-background contrast, sensor performance, and caused by atmospheric attenuation [21-23].

Also, thermal imagery is difficult to interpret and process with there is an existence of moisture absorption in the atmosphere. Normally, all objects emit infrared energy or electromagnetic energy at the speed of light as thermal heat. These electromagnetic energies are radiated in forms of waves with electrical and magnetic properties. Using a thermal imaging technology reveals that are not visible to the naked eye. Moreover, these thermal imageries can capture thermal images without making direct contact with the sample being tested. Therefore, the image being tested can capture thermal details at a safe distance and have a better chance of seeing temperature anomalies under normal operating conditions.

The thermal image is associated with different characteristics based on the IR radiation. These characteristics are absorptivity, emissivity, transmissivity, and reflectivity of infrared radiations [24] and the effects vary differently for every objects/material. Kirchhoff's law stated that the relationship between these characteristics as shown in (1)

Where $\eta$ is the absorptivity, $\varsigma$ is reflectivity and $\xi$ is for transmissivity [25]. During thermal equilibrium of an object, the absorption is equal to emission. For opaque objects equation can be expressed as (2)

$$
\eta+\varsigma=1 \text { or } \psi+\varsigma=1
$$

Where $\psi$ is the emissivity of the object.
Absorption of materials, thermal energy transmission, the intensity of emission, and the reflection of radiation are some factors affecting the thermal properties of the sample object to be measured. Figure 2 shows the factors that affect the sample object. The infrared sensors in thermal camera receive the total IR radiation emitted from the object's surface is directly related to the object's emissivity and its temperature, and define as the Stefan-Boltzmann Law [26-27] as shown in (3)

$$
\mathrm{E}=\sigma \psi \mathrm{T}^{4}
$$

Where $\mathrm{E}$ is the total amount of emitted radiation in an object per square meter, $\sigma$ is the Stefan-Boltzmann constant as $5.67 \times 10^{-8}$ $\frac{W}{m^{2} K^{4}}$ and $\mathrm{T}$ is designated for the temperature and measured in Kelvin $(\mathrm{K})$. Therefore, the object's temperature can determine if the emissivity of the object and total radiation emitted are known.

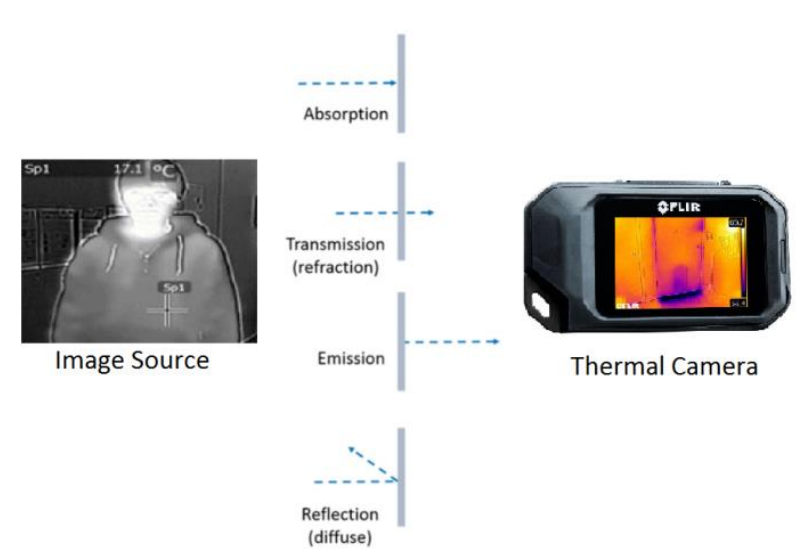

Fig. 2: Notations of the Factors Affecting the Thermal Properties of the Sample Image.

Digital image processing methods can improve the image information for further analysis and processing appropriate for data storage, transmission mode, and representation for automated machine recognition. Numerous algorithms are available were developed to enhance a more accurate image information [28-30] using digital image processing for different applications. Reference [31] describes one thermal image enhancement application using the inverted Otsu method. This work used an enhanced color mapping algorithm with inverted Otsu method however, it has no thresholding process for object segmentation and does not characterize the enhanced thermal image.

The thresholding algorithms presented in [32] proves that no single algorithm can enhance a thermal image. Also, the paper that is presented in [33] shows the selected thresholding methods from various categories and compared to measure the performances for different applications. However, no specific method was concluded which is appropriate for thermal image application. While in [34] presented a method for image thresholding and it was simulated in MATLAB. But it has no detailed description of the iteration process. The result in edge mapping which is presented in paper [35] uses edge direction and magnitude is far better against in using the edge magnitude alone.

Lastly, various papers presented with specific applications. In [36] discussed an algorithm for face detection utilizing thermal images, however, this paper has no actual hardware set-up and simulation. Reference [37] application is for thermal imaging of electrochemical power systems. This paper describes the related techniques of a thermal image for electrochemical power systems with the primary focus on fuel cells and batteries. Moreover, reference [38] is dedicated for analyzing a thermal image in the medical field.

This proposed algorithm was compared with stationary wavelet transform and to the morphology technique. The stationary wavelet transform has been used in several images processing applications. The SWT transform is applied to the image for edge enhancement 
and this method does not decompose an image, the sub-bands will have the same size as the input image [39]. On the other hand, morphological image processing deals with the shape of features in an image [40]. The morphological operations are used to remove imperfections which were introduced during segmentation.

Moreover, there several characteristics of thermal images based on its applications. The three most important are listed below.

1) The response to the temperature of an object, as shown in equation (4), the radiance of an object is a function of its temperature. This equation describes the relationship is known as Planck's equation.

$$
\mathrm{W}(\lambda, \mathrm{T})=\mathrm{e}(\lambda) \frac{c_{1}}{\pi \lambda^{5}}\left[\exp \left(\frac{c_{2}}{\lambda T}\right)-1\right]
$$

Where $\mathrm{W}(\lambda, \mathrm{T})$ is the radiance for a wavelength $\lambda$ and measures in $\mu \mathrm{m}$ while $\mathrm{T}$ stands for temperature in $\operatorname{Kelvin}, \mathrm{e}(\lambda)$ is the emissivity, $c_{1}$ and $c_{2}$ are the constants for this Planck's equation. The radiance is given in units of watts $\mathrm{m}^{-2}$ steradians ${ }^{-1} \mu \mathrm{m}$ [41]. Therefore, a thermal imager (thermal camera) can be used as a means of measuring the object's temperature and can provide a two-dimensional distribution graph between the relative and actual temperature which is appropriate for different types of applications.

2) The passive imaging is one of the important characteristics because there is no required external source of radiation is needed, the result provides a means of seeing at night-time, or even in poor illuminations.

3) Wavelength plays a vital part in visuality.

With these above characteristics, features like edges, shape, and size of the hottest regions can help to determine, characterize and detect the abnormalities of the subjected thermal images. Thus, this approach can play a significant role in many applications. Further enhancement and image processing can be employed to characterize the detected regions or segment for analyzations. Consequently, image processing plays a vital role in thermal imaging applications. Enhancing or developing an algorithm for thermal images can provide more reliable and accurate imaging method.

\section{Proposed algorithm}

This proposed algorithm aims to achieve a more vivid and sharp edge of thermal images for characterization. Figure 3 shows the proposed algorithm, the original input thermal image will be fed into three different channel (K-means clustering, inverted Otsu and high value thermal threshold processor), the resulting thermal images from K-means clustering and inverted Otsu will be subjected to Canny edge detection to extract some useful information (characteristics) from different point of visibilities and minimize the amount of data (information) to be processed. Then, the red color mapping will extract the red portion from the output of the highvalue thermal threshold. And all three outputs are combined together using a summing component.

To validate and verify the proposed algorithm, it was synthesized using the Xilinx Zynq 7000 ZC702 board. And, to manage the testing and simulation, an actual set-up was constructed to link with the Xilinx board. The set-up configuration is shown in Figure 4, it includes a thermal camera, FPGA board, and pc for configurations, etc. Also, Table 1 shows the details of the selected tools used for experimental testing. Additionally, different sample images were simulated using the three different techniques (stationary wavelet transform, the morphological technique, and the proposed algorithm) for comparison purposes.

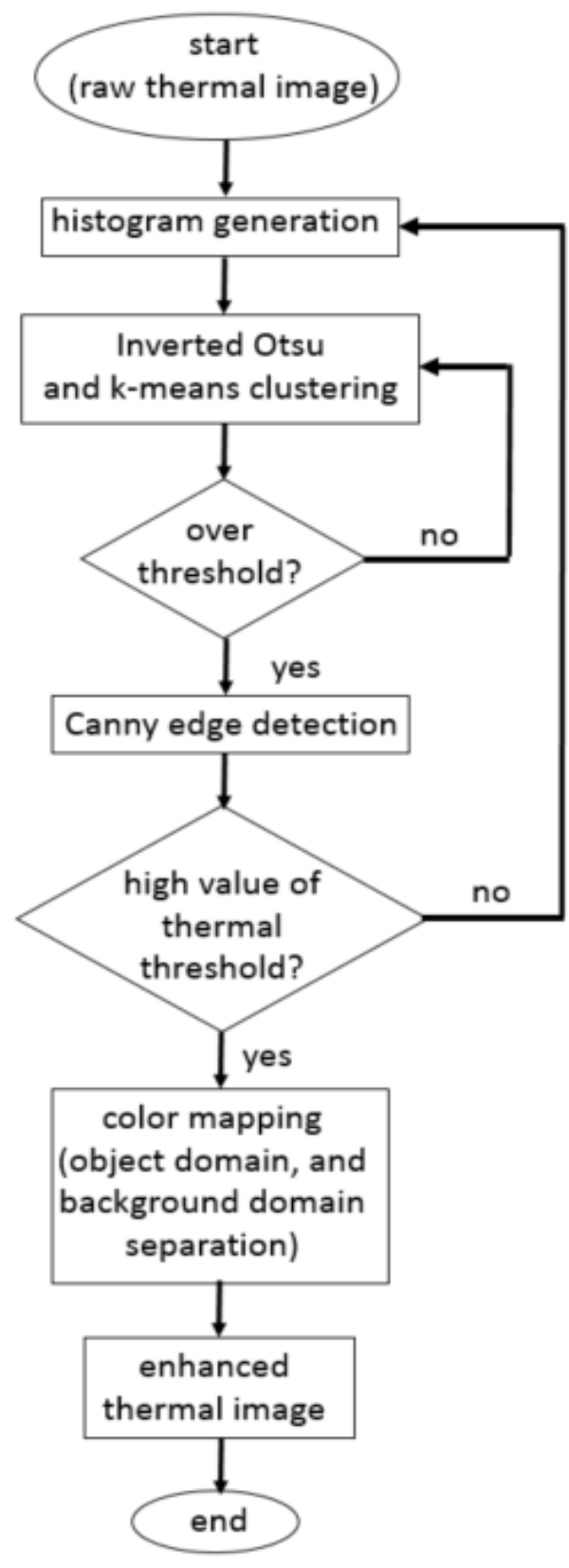

Fig. 3: Proposed Algorithm.

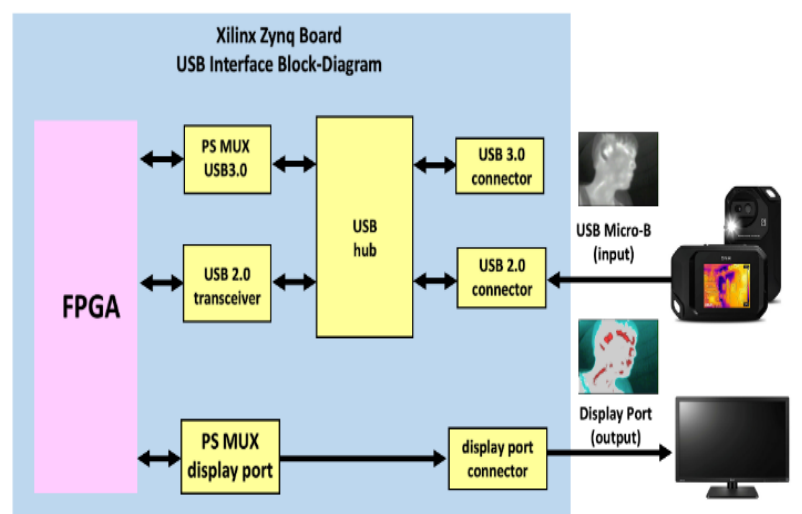

Fig. 4: Hardware Set-Up of the Proposed Algorithm.

Table 1: Selected Tools Used for Testing

\begin{tabular}{ll}
\hline Software Test tool & Visual studio, openCV 2.4.11 \\
\hline Hardware test tool & $\begin{array}{l}\text { Vivado 2017.2 (the complete design is a } \\
\text { parallel interface based on ARM and }\end{array}$ \\
& $\begin{array}{l}\text { FPGA) } \\
\text { Simulation tool }\end{array}$ \\
Isim
\end{tabular}




\section{Discussion and analysis of results}

To facilitate the efficiency of the proposed method, a comprehensive experimental analysis is conducted. The experimental set-up uses different images which are analyzed to extract important features, object's characteristics, and to identify any noise. If in case of discrepancy between the required ranges of the threshold, the misclassified pixels or frame is processed in order to enhance its quality. Also, this algorithm involves noise reduction that augments the signal to noise ratio during the radiation exposure, then to highlight some features which are not visible in the original images, contrast balance is used. Finally, edge detection method aims to define the discontinuities in the frame for consideration. Then, to illustrate the performance of the three different methods, the different images were simulated using SWT, Morphology and the proposed algorithm to verify which among the three methods can have a good result.

The experimental results show that the output image using the proposed algorithm outperforms the other techniques in a number of important features. To assess the perceptual quality of the sample image, various features could be estimated that may represent subjective quality which characteristics could be considered as desired or unwanted. Table 2 shows the tabulated comparison between the three methods in terms of edge visibility, contrast, and brightness.

Table 2: Comparison of Thermal Object Characteristics Results Using the Three Methods

\begin{tabular}{llll}
\hline $\begin{array}{l}\text { Thermal object } \\
\text { characteristics }\end{array}$ & Method & Morphology & Proposed \\
\hline edge visibility & partially visible & partially visi- & detecta- \\
object to back- & Ambiguous (no & partially dis- & ble \\
ground contrast & difference) & tinct & distinct \\
object brightness & almost dull & vague & $\begin{array}{l}\text { noticea- } \\
\text { ble }\end{array}$ \\
\hline
\end{tabular}

The results under the SWT method shows an ambiguity or almost unrecognizable to differentiate between the object and its background. While in the Morphology scheme, reveals partially visible the edge and contrast, but the object brightness is hazy. However, if this proposed algorithm is implemented the three thermal object characteristics are all noticeable. It shows that the enhanced thermal image by the applied algorithm has a more visible appearance and thermal object characteristics are more visually recognizable. The enhanced thermal image by the applied algorithm has a more visible appearance and thermal object characteristics are more visually recognizable. Compared to the other methods, the image enhanced by the algorithm maintains a better contrast, displays a higher brightness and colors to make a distinction between the object and its background.

The results show in Figure 5 are the thermal images tested during simulation of the proposed algorithm. These images provide a sharper edges making it more readily recognizable and show the outlines and boundaries between the object and its background. Relative to this, the resulting image emphasizes the thermal regions of the object as shown by the red area without losing its visibility. To further justify the performance of the proposed method under noisy, certain thermal images are contaminated by Gaussian noise and subjected to comparison using the three methods (SWT, Morphology, and proposed algorithm). Figure 6 shows the thermal images corrupted by Gaussian noise in different noise variances. Each noise variance tests 20 images. Since the noise variance is a random process, the testing repeats the process in 10 times and average the results

Table 3 shows the operating time of the three methods simulated to verify the three methods. Table 4 shows the resource utilization using this FPGA-based implementation, it shows that the designed memory architecture is lower than the two other methods.

Table 3: Processing Time Using the Three Methods

\begin{tabular}{lll}
\hline Methods & Software CPU (i7 GHz), RAM (2,400 MHz) & $\begin{array}{l}\text { Hardware } \\
\text { Simulation clock Frequency: 500 MHz }\end{array}$ \\
\hline SWT & $166 \mathrm{~ms}$ & $0.456 \mathrm{~ms}$ \\
Morphology & $172 \mathrm{~ms}$ & $0.288 \mathrm{~ms}$ \\
Proposed & $158 \mathrm{~ms}$ & $0.322 \mathrm{~ms}$ \\
\hline
\end{tabular}

(A)

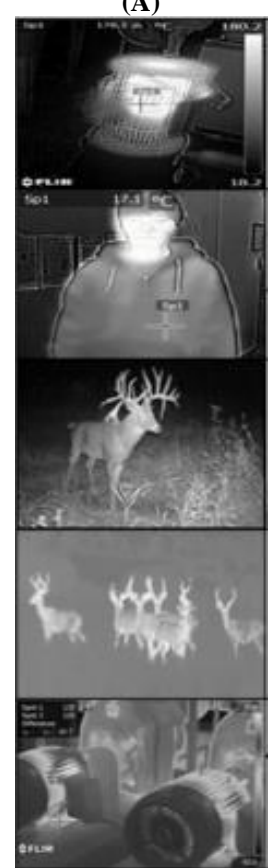

(B)

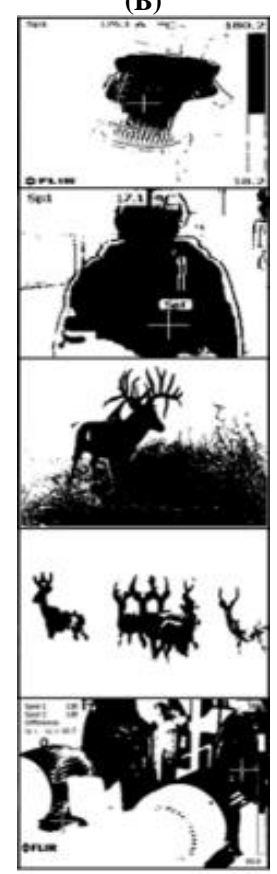

(C)

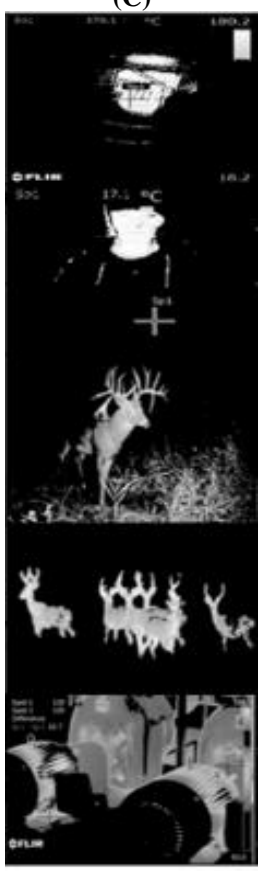

(D)

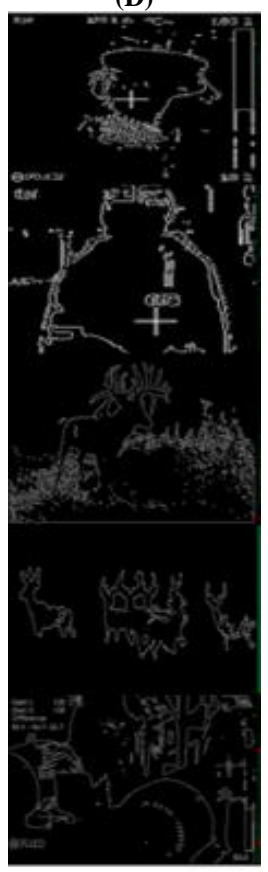

(E)

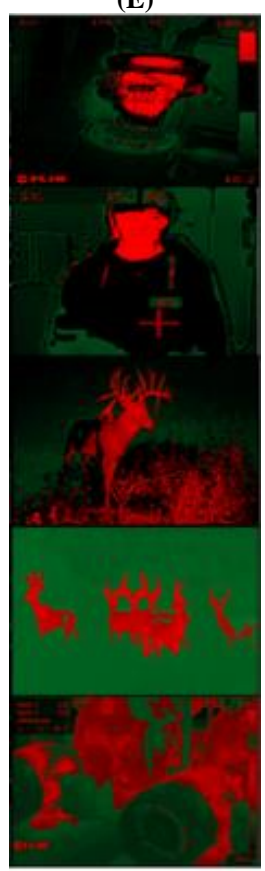

Fig. 5: (A) Original Image, (B) Integrated K-Means Clustering and Inverted Otsu Thresholding Image, (C) Heat Source Image, (D) Canny Edge Detection Image, (E) Image Results. 
misclassified pixels

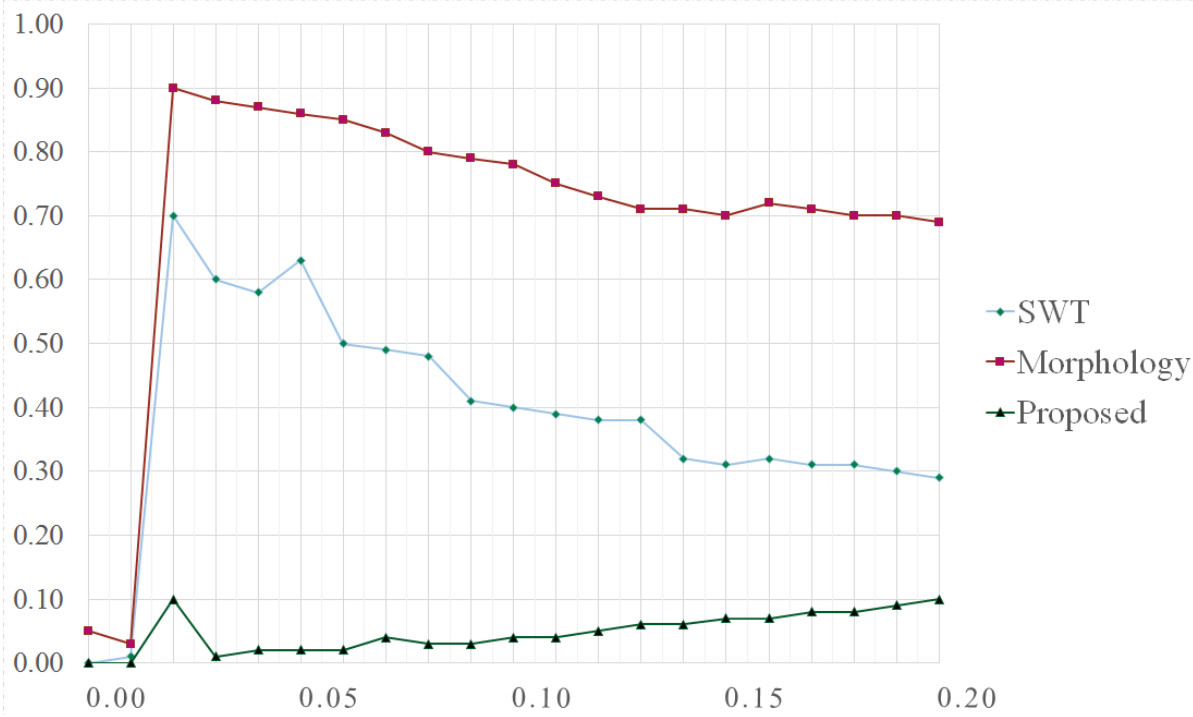

noise variance intensity

Fig. 6: Comparison of Thermal Images Corrupted by Gaussian Noise in Different Noise Variances Using the Three Methods (SWT, Morphology, and Proposed Algorithm).

Table 4: Resource Utilization of SWT, Morphology, and Proposed Scheme

\begin{tabular}{|c|c|c|c|c|}
\hline Logic & SWT & Morphology & Proposed & Available \\
\hline Number of slice & 582 & 416 & 418 & 19,200 \\
\hline Number of 4 input LUTs & 388 & 400 & 384 & 19,200 \\
\hline $\begin{array}{l}\text { Number of fully used } \\
\text { LUT-FF pairs }\end{array}$ & 402 & 622 & 352 & 44,977 \\
\hline Number of bonded IOBs & 24 & 30 & 28 & 220 \\
\hline $\begin{array}{l}\text { Number of Block } \\
\text { RAM/FIFO }\end{array}$ & 2 & 2 & 2 & 32 \\
\hline
\end{tabular}

\section{Conclusion}

The proposed algorithm aims the targets in terms of edge and boundary segmentation between the sampled object to its background. The image result after the proposed method is more robust to noise. Also, it minimizes the misclassified pixels when subjected to contamination like the Gaussian noise variance. Moreover, the original thermal regions on the sampled objects are naturally difficult to identify and almost invisible to the human eyes, but using the proposed algorithm, it can be easily detected in the enhanced image result. Furthermore, the presented results show a clear improvement in processing time, and the hardware utilization is reduced compared to SWT and Morphology techniques.

Lastly, this paper contributed to the development of in thermal images in visual recognition category and can be applied in healthcare, image retrieval, and data mining, especially in the non-destructive testing applications.

\section{References}

[1] Meng X, Applications and Research of Infrared Thermal Imaging Hazard Identification Technology, Proceeding of 2011 International Conference on Materials for Renewable Energy and Environment (ICMREE), (2011), pp: 1912-1916. https://doi.org/10.1109/ICMREE.2011.5930711.

[2] Gade R, Moeslund T, Nielsen SZ, Skov-Peterson H, Andersen HJ, Basselbjerg K, Dam HT, Jensen O, Jorgensen A, Lahrmann H, Madsen T, Bala ES, \& Povey B, Thermal Imaging Systems for Real-Time Applications in Smart Cities, International Journal of Computer Applications in Technology, Vol. 53, No. 4, (2016), pp:290-308. https://doi.org/10.1504/IJCAT.2016.076790.

[3] Endres F, Hess J, and Sturm J, 3-D Mapping with an RGB-D Camera", IEEE Transactions on Robotics, Vol. 30, No. 1, (2014), pp: 177-187. https://doi.org/10.1109/TRO.2013.2279412.
[4] Zhou Z, Malone E, Sato dos Santos G, Li N, Xu H, and Holder D, Comparison of Different Quadratic Regularization for Electrical Impedance Tomography, Proceeding of $6^{\text {th }}$ Conference of the International Federation for Medical and Biological Engineering, (2015), pp:200-203.

[5] Lee SU, Chung SY, and Park RH, A Comparative Performance Study of Several Global Thresholding Techniques for Segmentation, Computer Vision, Graphics, and Image Processing, Vol. 52, No. 2 , (1990), pp: 171-190. https://doi.org/10.1016/0734-189X(90)90053$\underline{\mathrm{X}}$

[6] Park CS, Kim HS, and Cha HW, Histogram Color Correction for Multi-View Cording, Proceeding of 2014 International SoC Design Conference (ISOCC), (2014), pp: 252-253. https://doi.org/10.1109/ISOCC.2014.7087633.

[7] Serfa Juan RO, and Kim HS, Reconfiguration of an FPGA-base Time-Triggered FlexRay Network Controller using EEDC, Journal of Circuits, Systems, and Computers, Vol. 27, No. 6, (2017), pp:111. https://doi.org/10.1142/S0218126618500962.

[8] Serfa Juan RO, and Kim HS, Implementation of EEDC for Trailer Segment in Enhanced FPGA-based FlexRay Controller, Journal of telecommunication, Electronic and Computer Engineering, Vol. 10, No. 1-9, (2018), pp:161-166.

[9] Mohd M, Hernan S, and Sharif Z, Application of K-means clustering in Hot Spot Detection for Thermal Infrared Images, Proceeding of 2017 IEEE Symposium on Computer Applications and Industrial Electronics, (2017), pp:107-110. https://doi.org/10.1109/ISCAIE.2017.8074959.

[10] Etehadtayakol M, Sadri S, and Ng EY, Application of K and Fuzzy $\mathrm{C}$-means for color segmentation of thermal infrared breast images, Journal of Medical Systems, Vol 34, No. 1, (2010), pp. 35-42. https://doi.org/10.1007/s10916-008-9213-1.

[11] Zhou H, Soh YC, and Wu X, Integrated analysis of CFD Data with K-means Clustering Algorithm and Extreme Learning Machine for Localized HVAC Control, Applied Thermal Engineering, Vol. 76 , (2015), pp: 98-104. https://doi.org/10.1016/j.applthermaleng.2014.10.004.

[12] Zhou S, Yang P, and Xie W, Infrared Image Segmentation Based on Otsu and Genetic Algorithm, Proceeding of 2011 International Conference on Multimedia Technology, (2011), pp: 5421-5424. https://doi.org/10.1109/ICMT.2011.6003109. 
[13] Heriansyah R, and Abu-Bakar S, Defect Detection in Thermal Image using Thresholding Technique, Proceeding of $6^{\text {th }}$ WSEAS International Conference on Circuits, Systems, Electronics, Control and Signal Processing, (2017), pp:341-346.

[14] Vala H, and Baxi A, Review on Otsu Image Segmentation Algorithm, International Journal of Advanced Research in Computer Engineering and Technology, Vol. 2, No. 2, (2013), pp. 387-389.

[15] Tzeng C, Yang Z, and Tsai W, Adaptive Data Hiding in Palette Images by Color Ordering and Mapping with Security Protection, IEEE Transactions on Communications, Vol. 54, No. 5, (2004), pp:791800. https://doi.org/10.1109/TCOMM.2004.826379.

[16] Faridul HS, Pouli T, and Chamaret C, A Survey of Color Mapping and its Applications, Proceeding of Eurographics 2014, (2014), pp: $1-25$.

[17] Toet A, and Walraven J, New false color mapping for image fusion, Optical Engineering, (1996), pp: 650-658. https://doi.org/10.1117/1.600657.

[18] Hogervirst M, and Toet A, Improved Color Mapping Methods for Multiband Nighttime Image Fusion, Journal of Imaging, Vol. 3, No. 3, (2017), pp:1-25.

[19] Kumar S, and Mahto D, Recent Trends in Industrial and Other Engineering Applications of Non-Destructive Testing: A Review, International Journal of Scientific and Engineering Research, Vol. 4, No. 9, (2013), pp. 183-195.

[20] Dua, G and Mulaveesala R, Aperiodic Thermal Wave Imaging Approach for Non-Destructive Testing and Evaluation of Steel Material: A Numerical Study, Journal of Nanoengineering and Nanomanufacturing, Vol. 6, No. 4, (2016), pp. 265-269. https://doi.org/10.1166/jnan.2016.1292.

[21] Fuliful F, Water Vapor Effect on 3-5 $\mu \mathrm{m}$ Band Thermal Imaging in Karbala City, Journal of Kerbala University, Vol. 12, No. 1, (2014), pp:184-191.

[22] Jacobs P, Thermal Infrared Characterization of Ground Targets and Backgrounds, $2^{\text {nd }}$ Edition, SPIE Press Book, 2006. https://doi.org/10.1117/3.651915.

[23] Ball M, and Pinkerton H, Factors Affecting the Accuracy of Thermal imaging Cameras in Volcanology, Journal of Geophysical Research, Vol. 111, No. 11, (2006), pp. 1-14. https://doi.org/10.1029/2005JB003829.

[24] Dey, N, Ashour A, and Althoupety A, Thermal Imaging in Medica Science, Recent Advances in Applied Thermal Imaging for Industrial Applications, (2017), pp: 1-20. https://doi.org/10.4018/978-15225-2423-6.ch004.

[25] Robitaille PM, On the Validity of Kirchhoff's Law of Thermal Emission, IEEE Transmissions on Plasma Science, Vol. 31, No. 6, (2003), pp:1263-1267. https://doi.org/10.1109/TPS.2003.820958.

[26] Paul H, Greenberger DM, Stenholm ST, and Schleich WP, The Stefan-Boltzmann Law: Two Classical Laws Give a Quantum One, Physica Scripta, (2015), pp:1-7. https://doi.org/10.1088/00318949/2015/T165/014027.

[27] Narimanov E, and Smolyaninov I, Beyond Stefan-Boltzmann Law: Thermal Hyper-Conductivity, CLEO Technical Digest, (2012), pp: $1-2$.

[28] Kumar A, and Santhi V, A Review on Geometric Invariant Digital Image Watermarking Techniques, International Journal of Computer Applications, Vol. 12, No. 9, (2011), pp. 31-36.

[29] Ashour A, Samanta S, Dey N, Kausar N, Abdessalemkaraa WB, and Hassanien AE, Computed Tomography Image Enhancement Using Cuckoo Search: A Log Transform Based Approach, Journal of Signal and Information Processing, (2015), pp. 244-257.

[30] Ghosh A, Sarkar A, Ashour A, Blas-Timar D, Dey N, and Blas V, Grid Color Moment Features in Glaucoma Classification, International Journal of Advanced Computer Science and Applications, Vol. 6, No. 9, (2015), pp:99-107.

[31] Ko BH, and Kim HS, Using Enhanced-Color Mapping Algorithm for Object Boundary Segmentation, International Journal of Applied Engineering Research, Vol 12, No. 15, (2017), pp. 5187-5190.

[32] Leedham G, Tan C, Takru K, Tan JHN, and Mian L, Comparison of Some Thresholding Algorithms for Text/Background Segmentation in Difficult Documents Images, Proceeding of Seventh International Conference on Document Analysis and Recognition,(2003), pp:1-6. https://doi.org/10.1109/ICDAR.2003.1227784.

[33] Sezgin M, Sankur B, Survey Over Image Thresholding Techniques and Quantitative Performance evaluation, Journal of Electronic Imaging, Vol. 13, No. 1, (2004), pp:46-164.

[34] Vijay PP, and Patil NC, Gray Scale Image Segmentation using Otsu thresholding Optimal Approach, Journal for Research, Vol. 2, No. 2, (2016), pp:20-24.
[35] Zhou Q, Li Z, and Aggarwal JK, Boundary Extraction in Thermal Images by Edge Map, Proceeding of 2004 ACM Symposium on Applied Computing, (2004), pp: 254-258. https://doi.org/10.1145/967900.967956

[36] Cheong YK, Yap VV, and Nisar H, A Novel Face Detection Algorithm using Thermal Imaging, Proceeding of IEEE 2014 Symposium on Computer Applications and Industrial Electronics, (2014) pp:208-213. https://doi.org/10.1109/ISCAIE.2014.7010239.

[37] Robinson J, Shearing P, and Brett D, Thermal Imaging of Electrochemical Power Systems: A Review, Journal of Imaging, Vol. 2, No. 1, (2016), pp:1-20, 2016. https://doi.org/10.3390/jimaging2010002.

[38] Ring EF, and Ammer K, Infrared Thermal Imaging in Medicine, Physiological Measurement Journal, Vol. 38, (2017), pp: 33-46.

[39] Demirel H, and Anbarjafari G, Image Resolution Enhancement by using Discrete and Stationary Wavelet Decomposition, IEEE Transactions on Image Processing, Vol. 20, No. 5, (2011), pp:1458-1460. https://doi.org/10.1109/TIP.2010.2087767.

[40] Goyal, M, Morphological Image Processing, International Journal of Computer Science and Technology, Vol. 2, No. 4, (2011), pp: 161165. Williams T, Thermal Imaging Cameras, Characteristics and Performance, CRC Press Taylor and Francis Group, 2009. https://doi.org/10.1201/9781420071863. 